\title{
Allometry displayed in the case of response to single exposure to arsine and its use in the extraction of the $0 \%$ lethal level
}

\author{
A. Guenda \\ Diffusion \& Implantation Group, Micro Electronic Clean Room, \\ CDTA, Algeria
}

\begin{abstract}
The relation between the exposure response to toxic gases, such as arsine, and the exposure time is almost non linear in a large domain of time. This non linear behaviour is especially visible when closer to the $0 \%$ lethal level, or closer to the $100 \%$ lethal level. An allometric behaviour has been displayed between the linear extrapolated exposure time corresponding to the $0 \%$ lethal level and the time corresponding to the medium of the linearity domain at a given concentration. Different methods have been used when displaying this allometry in order to ensure validity and reliability.

Keywords: single exposure, arsine concentration, exposure time, lethal level, $\mathrm{LCO} \%$, fitting, data processing, linearity domain, tangential extrapolated $\mathrm{LC} O \%$, allometry.
\end{abstract}

\section{Introduction}

Several approaches have been tested for the determination of the LC0\% from the experimental data mentioned by Levvy $[1,2]$. In order to conduct a coherent methodology in our investigations, it has been necessary to use a preliminary to filter and process the data, since these data were truncated.

It appears from the table given by Levvy that the author has build a kind of Noah's ark skeleton. The data appears first as being concise, but then the most important information, such as the linearity range, has been summarized, and even if we adopt more advanced tools for data processing, the method adopted by the Porton team was most satisfactory for the Second World War period. 
The fact that the population of the test did not reach the convergence threshold does not invalidate the allometry observed when processing these data.

It was necessary first to fit the experimental data given in the reference paper, and then to check the accuracy of the LC50\% that Levvy obtained by linear interpolation.

The strength of the fitting tool has been instrumented to improve the safety borders by trying several distributions with the intention of keeping the best quality factor. Other considerations taken into count are detailed below.

\section{Particularities of the fitting tool and its limitations}

The tool used for data processing is Table Curve 2D. This tool has been selected for its richness with non linear distributions, and because it allows fitting with other functions rather than polynomial ones.

Special care should be observed when completing the data with zeros at concentrations bellow the $\mathrm{LC} 0 \%$ and with hundreds at times greater than the time, which marks the beginning of the $100 \%$ lethal level.

It is possible to use other fitting tools that work only with polynomial functions after correcting the proceeded data at singularities, and refitting for a second time, but it should be pointed out that the order of polynomial functions used by the libraries of these tools are limited.

Another aspect to consider in the choice of the fitting tool is that for tools using only polynomial functions, the number of points in experimental data should be equal or greater than the order of the polynomial fitting function that will be selected by the tool when processing the data, so it is impossible to obtain a smooth fitting of data containing less than ten points. In our case, collections contain groups of five, four and three experimental points only.

\section{Exposure time - lethality response behaviour}

The wanted behaviour for a data group at a given concentration is somewhat similar to the obtained distribution for the Exposure - Lethality response curve at arsine concentration of $500 \mathrm{mg} / \mathrm{m} 3$, reproduced in Figure 1.

\section{Data processing methodology}

The methodology adopted for data processing at a $500 \mathrm{mg} / \mathrm{m} 3$ arsine concentration is as detailed below as an example of data processing.

First of all, the data should be completed by attributing $0 \%$ to times less than the maximum exposure time corresponding to the end of the $0 \%$ lethal level, and by attributing $100 \%$ lethal level to times greater than the time that marks the beginning of the $100 \%$ lethal level, so that the resulting curve has a quasi similar behaviour and the shape of a Pearson type IV distribution.

In the case of $500 \mathrm{mg} / \mathrm{m} 3$ arsine concentration, the experimental time marking the end of the $0 \%$ lethal level is $1,7 \mathrm{mn}$, and the time that marks the beginning of the $100 \%$ lethal level is $10 \mathrm{mn}$. This step of processing is detailed in the Table 1 , where the original and completed data have been reproduced. 


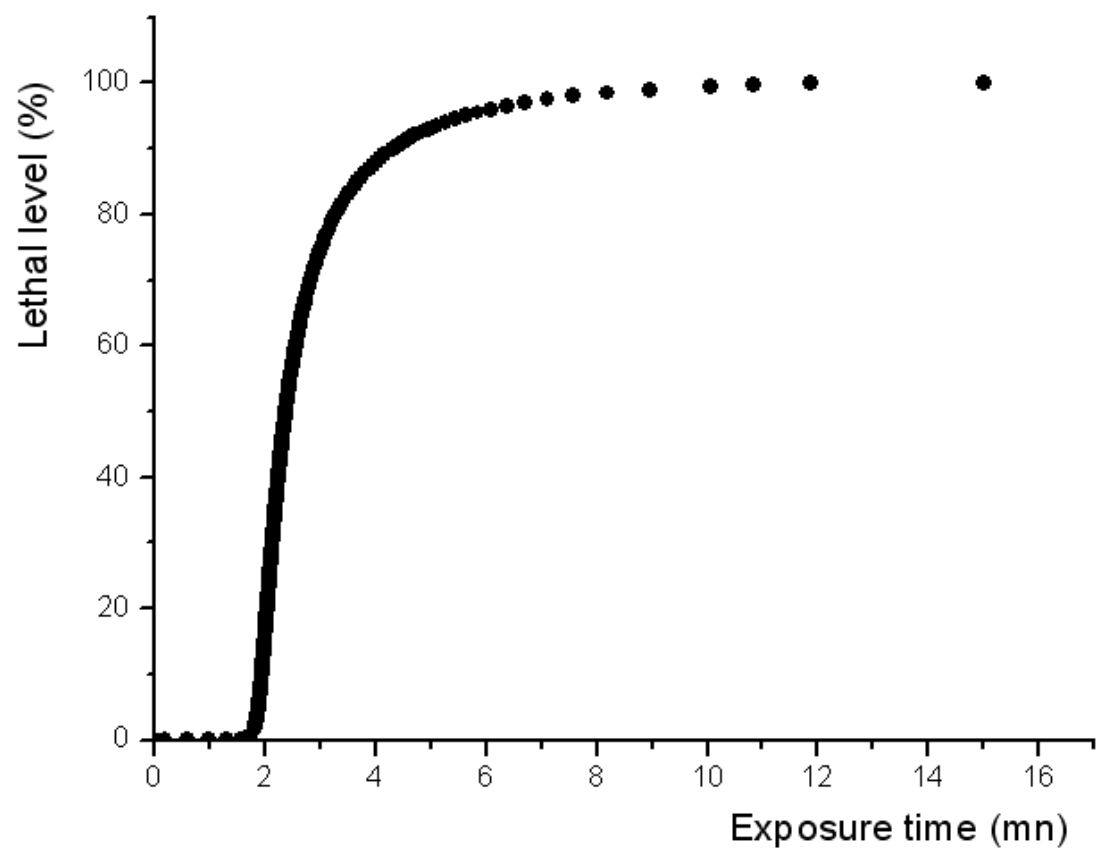

Figure 1: Time - lethality response at $500 \mathrm{mg} / \mathrm{m} 3$ based on Levvy [1].

Table 1: Completed set of time to lethality response at $500 \mathrm{mg} / \mathrm{m} 3$.

\begin{tabular}{|c|c|c|c|}
\hline \multicolumn{2}{|c|}{$\begin{array}{l}\text { Original data given by Levvy for } \\
500 \mathrm{mg} / \mathrm{m} 3\end{array}$} & \multicolumn{2}{|c|}{ Completed data for $500 \mathrm{mg} / \mathrm{m} 3$} \\
\hline $\begin{array}{c}\text { Exposure time } \\
(\mathrm{mn})\end{array}$ & Lethal level (\%) & $\begin{array}{l}\text { Exposure time } \\
(\mathrm{mn})\end{array}$ & Lethal level (\%) \\
\hline blank & blank & 0 & 0 \\
\hline blank & blank & 1,1 & 0 \\
\hline blank & blank & 1,5 & 0 \\
\hline 1,7 & 0 & 1,7 & 0 \\
\hline 2,5 & 57 & 2,5 & 57 \\
\hline 5 & 93 & 5 & 93 \\
\hline 10 & 100 & 10 & 100 \\
\hline blank & blank & 12 & 100 \\
\hline blank & blank & 15 & 100 \\
\hline blank & blank & 20 & 100 \\
\hline blank & blank & 50 & 100 \\
\hline
\end{tabular}


Table 2: $\quad$ Completed data at $2500 \mathrm{mg} / \mathrm{m} 3$ arsine concentration.

\begin{tabular}{|c|c|c|c|}
\hline \multicolumn{2}{|c|}{$\begin{array}{l}\text { Original data given by Levvy for } \\
\qquad 2500 \mathrm{mg} / \mathrm{m} 3\end{array}$} & \multicolumn{2}{|c|}{ Completed data for $2500 \mathrm{mg} / \mathrm{m} 3$} \\
\hline $\begin{array}{c}\text { Exposure time } \\
(\mathrm{mn})\end{array}$ & Lethal level (\%) & $\begin{array}{l}\text { Exposure time } \\
(\mathrm{mn})\end{array}$ & Lethal level (\%) \\
\hline blank & blank & 0 & 0 \\
\hline blank & blank & 0,05 & 0 \\
\hline 0,33 & 20 & 0,33 & 20 \\
\hline 0,5 & 93 & 0,5 & 93 \\
\hline blank & blank & 0,65 & 100 \\
\hline
\end{tabular}

Table 3: Completed data at $1000 \mathrm{mg} / \mathrm{m} 3$ arsine concentration.

\begin{tabular}{|c|c|c|c|}
\hline \multicolumn{2}{|c|}{$\begin{array}{c}\text { Original data given by Levvy for } \\
1000 \mathrm{mg} / \mathrm{m} 3\end{array}$} & \multicolumn{2}{c|}{ Completed data for 1000mg/m3 } \\
\hline $\begin{array}{c}\text { Exposure time } \\
(\mathrm{mn})\end{array}$ & Lethal level (\%) & $\begin{array}{c}\text { Exposure time } \\
(\mathrm{mn})\end{array}$ & Lethal level (\%) \\
\hline blank & blank & 0 & 0 \\
\hline 0,83 & 13 & 0,83 & 13 \\
\hline 1,25 & 57 & 1,25 & 57 \\
\hline blank & blank & 7,5 & 100 \\
\hline
\end{tabular}

Table 4: $\quad$ Completed data at $250 \mathrm{mg} / \mathrm{m} 3$ arsine concentration.

\begin{tabular}{|c|c|c|c|}
\hline \multicolumn{2}{|c|}{$\begin{array}{l}\text { Original data given by Levvy for } \\
\qquad 250 \mathrm{mg} / \mathrm{m} 3\end{array}$} & \multicolumn{2}{|c|}{ Completed data for $250 \mathrm{mg} / \mathrm{m} 3$} \\
\hline $\begin{array}{c}\text { Exposure time } \\
(\mathrm{mn})\end{array}$ & Lethal level (\%) & $\begin{array}{l}\text { Exposure time } \\
(\mathrm{mn})\end{array}$ & Lethal level (\%) \\
\hline blank & blank & 0 & 0 \\
\hline blank & blank & 1 & 0 \\
\hline blank & blank & 2 & 0 \\
\hline 9 & 33 & 9 & 33 \\
\hline 15 & 70 & 15 & 70 \\
\hline blank & blank & 40 & 100 \\
\hline blank & blank & 50 & 100 \\
\hline blank & blank & 100 & 100 \\
\hline
\end{tabular}

For the other groups of the data set, the completed data are summarized in Tables 2-4.

As a remark, it should be noted that completing the data summarized in Tables 2-4 and their use in fitting curves has been possible only because there are certainties about the exposure response and about the behaviour of the associated distributions. 
Table 5: $\quad$ Completed data at $100 \mathrm{mg} / \mathrm{m} 3$ arsine concentration.

\begin{tabular}{|c|c|c|c|}
\hline \multicolumn{2}{|c|}{$\begin{array}{l}\text { Original data given by Levvy for } \\
\qquad 100 \mathrm{mg} / \mathrm{m} 3\end{array}$} & \multicolumn{2}{|c|}{ Completed data for $100 \mathrm{mg} / \mathrm{m} 3$} \\
\hline $\begin{array}{l}\text { Exposure time } \\
(\mathrm{mn})\end{array}$ & Lethal level (\%) & $\begin{array}{l}\text { Exposure time } \\
\text { (mn) }\end{array}$ & Lethal level (\%) \\
\hline blank & blank & 1 & 0 \\
\hline blank & blank & 2 & 0 \\
\hline blank & blank & 3 & 0 \\
\hline blank & blank & 4 & 0 \\
\hline blank & blank & 5 & 0 \\
\hline blank & blank & 6 & 0 \\
\hline blank & blank & 7 & 0 \\
\hline blank & blank & 8 & 0 \\
\hline blank & blank & 9 & 0 \\
\hline blank & blank & 10 & 0 \\
\hline 50 & 50 & 50 & 50 \\
\hline 70 & 100 & 70 & 100 \\
\hline blank & blank & 75 & 100 \\
\hline blank & blank & 80 & 100 \\
\hline blank & Blank & 90 & 100 \\
\hline blank & blank & 100 & 100 \\
\hline
\end{tabular}

The case of Table 1 is simple to complete, since the $0 \%$ and $100 \%$ lethal levels have been experimentally given so that the completing operation consists only in associating $0 \%$ to times less than the experimental time associated to the $0 \%$ lethal level, and also in associating $100 \%$ to times larger than the experimental time associated to the experimental $100 \%$ lethal level.

For other cases where there is at least a threshold limit missing in the original data, a simple and roughly assumption has been made in order to get an approximate value.

As an example, in the case of Table 3, for situating an overestimated time associated to a $100 \%$ lethal level with certainty, we have made the assumption below:

- Arsine concentration: 500mg/m3 (from Table 1)

$$
\begin{aligned}
2,5 \mathrm{mn} & \rightarrow 57 \% \\
10 \mathrm{mn} & \rightarrow \quad 100 \%
\end{aligned}
$$

- Arsine concentration: 1000mg/m3 (from Table 3)

$$
\begin{aligned}
1,25 \mathrm{mn} & \rightarrow \quad 57 \% \\
\mathrm{Xmn} & \rightarrow \quad 100 \%
\end{aligned}
$$


- Assumption for overestimated time X:

$$
\frac{(X-1,25)}{1,25}=\frac{(10-2,5)}{2,5}
$$

The estimated time associated to with the $100 \%$ lethal level in the case of arsine concentration of $1000 \mathrm{mg} / \mathrm{m} 3$ is equal to $5 \mathrm{mn}$. It is possible to process by steps, for example by evaluating the associated time to the $93 \%$ lethal level, and so applying the same equality between ratios between the $93 \%$ and $100 \%$ differences.

The general equation form is:

$$
\frac{[X 2(k \%, C 1)-X 1(m \%, C 1)]}{X 1(m \%, C 1)}=\frac{\left[X " 2(k \%, C 2)-X^{" 1}(m \%, C 2)\right]}{X^{\prime \prime} 1(m \%, C 2)}
$$

where the different $\mathrm{Xj}$ are times associated to $\mathrm{k} \%$ and $\mathrm{m} \%$ lethal levels at $\mathrm{C} 1$ and $\mathrm{C} 2$ exposure concentrations.

As a margin of caution we added the double time associated to the reference level, which in this example is the $57 \%$ lethal level.

Thus, the final overestimated time is:

$$
\mathrm{X}(100 \% ; 1000 \mathrm{mg} / \mathrm{m} 3)=5+(2 \times 1,25) \mathrm{mn}=7,5 \mathrm{mn}
$$

\section{Influence of the margin of caution on the choice of lethality response distribution}

As a reminder, it should be noted that Levvy used only groups of thirty mice per group when establishing every lethality level at a given concentration. Since considerable doubt exists about reaching the convergence threshold, special care should be observed when selecting the distribution closer to the real response.

The lack of convergence is more evident for the drawing up of lethality levels at arsine exposure concentration of $25 \mathrm{mg} / \mathrm{m} 3$ where only groups of 06 mice per lethality level have been used.

Our adopted approach consists of selecting the more shifted distributions, which allow a lowering of the time corresponding to the beginning of the lethality, and lowering the time corresponding to the beginning of the $100 \%$ lethal level, even if the first automatic selected distributions are those corresponding to the best adjustment factors. The final selected distributions should not, however, exhibit a degraded adjustment factor.

\section{Allometry displayed}

An allometry character was first pointed out by Levvy when studying the ration of the amount of arsine absorbed by $\mathrm{kg}$ of body weight divided by the product of arsine concentration in air and time exposure. 
Since our interest is focused on the safe and accurate determination of the time above which the lethality begins, we proceeded to a comparison between the obtained time corresponding to this lethality limit threshold obtained by data processing after a fitting as detailed above, and the tangential extrapolated threshold time limit.

The tangential extrapolated threshold time has been obtained by two different but closely analogous methods.

The first method for determining the tangential extrapolated threshold time is the one which is situating the middle of linearity range from a given exposure arsine concentration. The method is focusing on the reduced relative error between values obtained by simple linear interpolation and the proceeded data obtained by fitting process for a given stride.

The relative error should be equal or less than a fixed value. In our case, we assumed that the relative error should be less than $1 \%$.

It should be noted the fact that the middle of the linearity range do not change much if we reduce or increase the tolerance. The middle of the linearity range

Table 6: Determination of the relative error between fitted lethal levels and linear extrapolated ones at an exposure arsine concentration of $2500 \mathrm{mg} / \mathrm{m} 3$ for a $5 \%$ stride.

\begin{tabular}{|c|c|c|c|}
\hline Fitted time (mn) & $\begin{array}{c}\text { Fitted lethal } \\
\text { level (\%) }\end{array}$ & $\begin{array}{c}\text { Linear } \\
\text { extrapolated } \\
\text { lethal level (\%) }\end{array}$ & $\begin{array}{c}\text { Relative Error } \\
\text { between linear } \\
\text { interpolation and } \\
\text { fitting }\end{array}$ \\
\hline 0,2710464 & 5 & Blank & Blank \\
\hline 0,29771134 & 10 & 10,97125817 & 9,712581748 \\
\hline 0,31570188 & 15 & 15,57178279 & 3,811885246 \\
\hline 0,33 & 20 & 20,38242246 & 1,912112306 \\
\hline 0,34226635 & 25 & 25,2686569 & 1,074627584 \\
\hline 0,35328174 & 30 & 30,19040551 & 0,634685037 \\
\hline 0,36348895 & 35 & 35,13114127 & 0,374689342 \\
\hline 0,37317441 & 40 & 40,08262481 & 0,206562021 \\
\hline 0,38254497 & 45 & 45,04002194 & 0,088937655 \\
\hline 0,39176671 & 50 & 50,00008404 & 0,000168084 \\
\hline 0,40098814 & 55 & 54,96015547 & $-0,072444595$ \\
\hline 0,41035772 & 60 & 59,91757316 & $-0,137378065$ \\
\hline 0,4200414 & 65 & 64,86908252 & $-0,201411513$ \\
\hline 0,43024582 & 70 & 69,809889 & $-0,271587148$ \\
\hline 0,4412569 & 75 & 74,73174172 & $-0,357677712$ \\
\hline 0,45351649 & 80 & 79,61818813 & $-0,477264834$ \\
\hline 0,46780322 & 85 & 84,42932219 & $-0,671385659$ \\
\hline 0,48577138 & 90 & 89,0314743 & $-1,076139669$ \\
\hline 0,51237292 & 95 & 91,619787 & $-3,558118947$ \\
\hline 0,65000001 & 100 & Blank & Blank \\
\hline
\end{tabular}


will change due to this change in allowed tolerance, but the value of the tangential extrapolated threshold time at $0 \%$ lethality level do not change significantly.

The second method consists on the localization of the lethality level which is corresponding to minimal relative error between the fitted lethal levels and those obtained by linear interpolation from these fitted values for a given stride.

As an example for arsine concentration of $2500 \mathrm{mg} / \mathrm{m} 3$ in air, Table 6 is summarizing the method to determine the reduced relative error and the tangential extrapolated threshold time.

When using the first method, it can be seen that the minimum of relative error is located around the $50 \%$ lethal level. When the stride is reduced to $1 \%$, the minimum of relative error is still located at $50 \%$ lethal level.

If the second method is used for a relative error less than $0,5 \%$, the linearity range is located between the lethal levels $35 \%$ and $80 \%$, and the medium of this range is around $57 \%$.

When enlarging the relative error tolerance to $1 \%$ for the same stride, the linearity domain is located between $90 \%$ and $25 \%$, and the middle of linearity range is still around $57 \%$.

Since these preliminary checks do not represent a confirmation of the validity and reliability of the method, the calculations made in table 6 above have been conducted a second time for a given stride of $2 \%$, and tolerated relative errors in range where they are less than $0,2 \%$, and less than $0,1 \%$. The middle of linearity domain has been located around the $57 \%$ lethal level.

The calculations have been also conducted for a given stride of $1 \%$ with three relative error tolerance limits. The tolerance limit of $0,5 \%$ lead to a linearity domain with a medium located at the $53 \%$ lethality level.

A tolerance limit of $0,2 \%$ lead to a linearity domain with a medium located at the $55 \%$ lethality level. The last value of tolerance limit generated a linearity domain with a medium located at the $57 \%$ lethality level.

At least, it is deducted from the discussion above that more reduced is the tolerance limit, better is the convergence of the medium value of the linearity domain.

For the first method this convergence aspect of the minimal relative error cannot be observed with only one given exposure concentration, even when using the change of the stride.

The minimal relative error method has been adopted for determining the tangential extrapolated threshold time. Since the time elapsed between the lethality level of $0,085 \%$ and the $1 \%$ lethality level is large, a set of three curves has been three allometry curves has been established. The first one is taking the $0,085 \%$ level obtained from fitted curves as being the $0 \%$ lethality level. The second curve is working with the $1 \%$ lethality level as being the real $0 \%$. The third one is taking the equally weighted level between $0,085 \%$ and $1 \%$ as being the real $0 \%$.

Table 7 summarizes the curve obtained with the assumed $1 \%$ lethal level as being the real $0 \%$ lethality level.

The obtained curve illustrated in figure 2 shows an evident allometry. 
Table 7: $\quad$ Relation between linear extrapolated time and fitted time at $0 \%$ lethality level.

\begin{tabular}{|c|c|c|c|c|}
\hline $\begin{array}{c}\mathrm{C} \\
(\mathrm{mg} / \mathrm{m} 3)\end{array}$ & $\begin{array}{c}\text { Lethal level } \\
\text { of minimal } \\
\text { relative } \\
\text { error (\%) }\end{array}$ & $\begin{array}{c}\text { Linear } \\
\text { extrapolated } \\
\text { time at } 0 \% \\
\text { lethal level X0 } \\
(\mathrm{mn})\end{array}$ & $\begin{array}{c}\text { Fitted time of } \\
1 \% \text { lethal } \\
\text { level X1 }(\mathrm{mn})\end{array}$ & $\begin{array}{c}(\mathrm{X} 0-\mathrm{X} 1) / \\
\mathrm{X} 0\end{array}$ \\
\hline 2500 & $50 \%$ & 0,29978321 & 0,22102784 & 0,262707741 \\
\hline 1000 & $35 \%$ & 0,73649185 & 0,56921966 & 0,227120219 \\
\hline 500 & $20 \%$ & 1,8632761 & 1,7834217 & 0,042856987 \\
\hline 250 & $35 \%$ & 4,20224565 & 3,0398476 & 0,276613541 \\
\hline 100 & $51 \%$ & 40,562328 & 28,083479 & 0,307646272 \\
\hline
\end{tabular}



Arsine Concentration $\left(\mathrm{mg} / \mathrm{m}^{3}\right)$

Figure 2: $\quad$ Observed allometry in the case of single exposure to arsine with a minimum at around $500 \mathrm{mg} / \mathrm{m} 3$.

\section{Conclusion}

Using an adequate fitting tool like Table Curve 2D combined to other simple tools like Origin and Microsoft Excel, it has been possible as a first step to draw the Time - Response curves at given concentrations with a set of restricted experimental data. As we have indicated, some of these data are containing only groups of four, three and sometimes only two experimental points. 
To establish a complete and general Time - Concentration - Response relationship, an unsuccessful attempt has been tried using a $3 \mathrm{D}$ fitting of the obtained by a two dimensions fitted data. Unfortunately, the $3 \mathrm{D}$ fitting tool is using only polynomial distributions which are not the recommended functions in a fitting process.

Several forms of allometry are observed in different fields of biology, like for animals, plants and humans $[3,4]$

The observed allometry between the real $0 \%$ lethal level and the projected $0 \%$ lethal level obtained by projection from linear domain can help in drawing up a general Time - Concentration - Response relationship, applied for single or repeated exposures. The methods adopted and explored when displaying this allometry can also be applied to exposure to other gases.

This allometry can be used to determine the time which marks the beginning of lethality, especially when studying or assessing arsine toxicity in the case of repeated exposure.

The lethal level $\mathrm{LC} 0 \%$ is of special interest, since it is the level which marks the beginning of the lethality. This level also represents the maximum value of the range of AEGL3 values, as defined by the EPA to humans. The determination of this level will allow people a subsequent revaluation of Acute Exposure Guideline Levels for humans.

The methods exposed in this paper can be applied to other gases exposure responses when taking in consideration the analogies and differences between the complex arsine action and the studied gas. As one of the response differences, one should remember the dead rate of lung, and the rate of absorbed arsine per body weight in each organ [1].

For arsine, the allometry has been explored only for the $0 \%$ lethal level, but it is possible to explore if this gas is showing also an allometry for the $100 \%$ lethal level. This $100 \%$ lethal level can be useful in the case of industrial catastrophes. Even if this scenario is less probable in the case of arsine, application of allometry analogy to other gases which appear as being less toxic but much frequently used in large quantities can help for first aid intervention in disaster area.

\section{References}

[1] Levvy G.A., A Study of Arsine Poisoning, August 1946.

[2] Fishbein, L., Arsine Human Health Aspects, Concise International Chemical Assessment Document 47, second draft, World Health Organization, Geneva, 2002.

[3] Schwartz, D., Les modèles en biologie et en médecine, Pour la Science magazine, n²27, September 1996.

[4] West, G.B., Brown, J.H. \& Enquist, B.J., The origin of universal scaling laws in biology, Oxford University Press, 2000. 